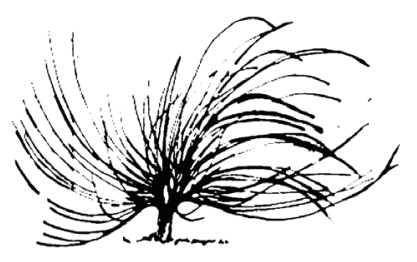

\title{
Proyecto Manejo básico de oficinas: Una intervención educativa para la formación de docentes de Educación Comercial
}

\author{
Herminia Ramírez Alfaro ${ }^{1}$ \\ Universidad Nacional \\ Heredia, Costa Rica \\ hminara@gmail.com \\ Ingrid Sánchez Alvarado ${ }^{2}$ \\ Universidad Nacional \\ Heredia, Costa Rica \\ ingriduna@gmail.com
}

\begin{abstract}
Resumen
La propuesta que se presenta tiene como objetivo evidenciar el aporte del proyecto Manejo básico de oficinas como intervención educativa que permite la articulación entre las áreas estratégicas de la Universidad Nacional de Costa Rica, en este caso específico, la docencia y la extensión, en la formación docente. Se proponen cambios innovadores en el modelo de formación de docentes, convirtiéndolo en un currículo flexible, más práctico, más vivencial, contextualizado y acorde con los requerimientos
\end{abstract}

\section{(C) (1) $\Theta \Theta$}

Recibido: 28 de marzo de 2017-Aprobado: 21 de mayo de 2018

http://dx.doi.org/10.15359/rep.13-1.8

1 Máster en Educación de la Universidad Nacional, Licenciada en Secretariado Profesional con Énfasis en Educación Comercial de la Universidad Nacional de Costa Rica, Académica de la División de Educología.

2 Máster en Psicopedagogía de la Universidad de La Salle, Licenciada en Secretariado Profesional con Énfasis en Educación Comercial de la Universidad Nacional de Costa Rica, Académica de la Escuela de Secretariado Profesional 
de estudiantes de Educación Comercial. Además, el modelo se enriquece al ampliar los espacios para realizar procesos de práctica y que los docentes en formación puedan ejercer su labor con diversas poblaciones jóvenes, adultas y adultas mayores. Un punto fundamental es cómo esta experiencia de formación docente se convierte, a la vez, en una intervención educativa, que busca por medio de la educación no formal brindar espacios de capacitación a grupos en desventaja social y con esto se favorece la posibilidad de superación personal, laboral y social. Por ello, se pretende mostrar la importancia de una iniciativa que ha permitido innovar el currículo de formación docente e intervenir por medio de la educación ante las necesidades sociales, mediante un proyecto de extensión y docencia.

Palabras clave: Formación docente, intervención educativa, docencia, extensión, modelo.

\begin{abstract}
The proposal presented by the Proyecto Manejo Básico de Oficinas makes evident the importance of coordination between the strategic areas of the Universidad Nacional of Costa Rica, in this specific case, teaching and university extension. Innovative changes are proposed in the model of teacher training, making it flexible, more practical, more experiential, contextualized and according to the requirements, both social and student of Education Comercial curriculum. Furthermore, the model is enriched to increase opportunities for practice processes and training teachers to carry out their work with diverse populations, from youth, adults and seniors. A key point is how this experience of teacher training becomes both an educational intervention, which seeks to provide training opportunities to people in social disadvantage through non-formal education, and it favors the possibility of overcoming it personally, socially and occupationally. Therefore, this article intends to show the importance of an initiative that has enabled an innovative teacher training curriculum and address social needs through education by means of an extension and teaching project.
\end{abstract}

Keywords: Teacher training, educative intervention, teaching, social extension, model 


\section{Introducción}

Desde su nacimiento, la Universidad Nacional de Costa Rica se ha constituido en una institución de educación superior que se ha caracterizado por su visión integral en los procesos formativos "desde sus orígenes, ha construido un proyecto educativo, científico, cultural y social integral, inclusivo y sobre todo, al servicio de la sociedad costarricense" (página web UNA, 2016).

Fiel reflejo de este papel fundamental se encuentra en los procesos de formación docente, tanto para educación primaria como para la educación secundaria, que es asumida y respaldada por el Centro de Investigación y Docencia en Educación (CIDE), el cual cuenta con tres divisiones encargadas de la formación inicial.

En el caso particular que se presenta, la carrera de Educación Comercial se orienta a la formación de docentes para colegios técnicos y está gestionada curricularmente por dos unidades académicas, la Escuela de Secretariado Profesional, que ofrece la parte disciplinar y la División de Educología, que se encarga del componente pedagógico.

Actualmente la sociedad vive cambios vertiginosos obligando a las instituciones de educación superior a buscar nuevas estrategias para tratar de responder las demandas sociales que van emergiendo. En este marco se ve inmerso el personal docente, como profesional de la educación, que contribuye en la formación de ciudadanía para el adecuado desenvolvimiento de su entorno.

Queda claro que el rol docente cambia y se requiere de una participación comprometida, incluso en alguna medida predictiva de situaciones futuras a las que se enfrentarán sus estudiantes. Es necesario, tal como lo plantean González y González (2007), que el personal docente pueda proponer situaciones de aprendizaje que favorezcan en el estudiantado la autonomía, la adquisición de conocimientos y el fortalecimiento de valores en espacios educativos participativos:

Para ello el docente ha de generar competencias didácticas, motivación y cierto compromiso profesional que le permitan desarrollar sus clases a través de metodologías participativas de enseñanza que posibiliten vincular la teoría con la práctica profesional en un contexto de diálogo, con el empleo de métodos y técnicas de evaluación que centren la atención en el estudiante como sujeto de aprendizaje. (p. 2) 
Por tanto, la formación de docentes debe ser integral, de manera que pueda propiciar la adquisición de nuevos conocimientos disciplinares, sociales y personales. Es hacia esta meta que las instituciones de educación superior deben dirigirse, hacia la consecución de uno de sus principales objetivos: formar profesionales que respondan a las necesidades de la sociedad, buscando alternativas de mejoramiento continuo, a través de procesos que validen la pertinencia con la realidad social y la búsqueda incesante del logro de respuestas acordes a las necesidades planteadas por los diversos contextos culturales, sociales y económicos.

La formación docente precisa, por lo tanto, de procesos actualizados e innovadores, que renueven las propuestas existentes, que le brinden al cuerpo docente una formación integral, con herramientas para asumir la responsabilidad que representa la educación.

En esta línea de pensamiento, existen diversos debates en cuanto a la formación de docentes, en lo que se denomina la "tensión entre lo disciplinar y lo pedagógico" (Progré, 2004); es decir, cómo lograr que exista un proceso integrado entre lo disciplinar y lo pedagógico. Esto genera una búsqueda de experiencias innovadoras en su formación, de manera que se deje de lado el énfasis en los contenidos para dar paso al desarrollo de conocimientos, habilidades y actitudes como un proceso integral de formación.

En Costa Rica, particularmente en la Universidad Nacional, en las carreras de la enseñanza secundaria, los modelos de formación docente no han sido evaluados de manera óptima, en procesos de autoevaluación y acreditación, por el mismo estudiantado graduado por la falta de una mayor relación entre la teoría - práctica; así como la carencia de un número más variado de prácticas durante el proceso de formación. Esta crítica nace del modelo de formación que, en un alto porcentaje de las carreras de la enseñanza se da, en el que las prácticas se desarrollan al finalizar la formación.

En cuanto a la formación de docentes, en la educación superior, esta engloba las más diversas temáticas: la particularidad misma del proceso de enseñanza y aprendizaje, sus actores, los diseños curriculares, los fundamentos pedagógicos, entre otros. En este caso particular, los ejes temáticos principales que se desarrollarán serán la formación de docentes, la experiencia de una intervención educativa en un modelo de formación, la relación docencia y extensión universitaria, y el esbozo de un modelo de formación docente que describe la relación dialéctica entre la teoría y la práctica. 


\section{La formación docente}

En primera instancia es importante conceptualizar el término formación, debido a que pueden surgir diversas acepciones según el campo en el que se pueda ubicar. De acuerdo con Godorokin:

El concepto de "formación" implica una acción profunda ejercida sobre el sujeto, tendiente a la transformación de todo su ser, que apunta simultáneamente sobre el saber-hacer, el saber-obrar y el saber pensar, ocupando una posición intermedia entre educación e instrucción. (2005, p. 2)

Lo anterior pretende establecer una relación del saber, la práctica, las representaciones y las identificaciones que forman a quien se está formando, esto tanto en el plano del conocimiento, como en lo afectivo, en lo social y lo práctico; es decir, se pretende orientar el proceso formativo de manera integral, más que en algo meramente teórico.

La formación docente debe garantizar que los grupos educadores logren procesos educativos efectivos y lleven a cabo experiencias de enseñanza y aprendizaje exitosas, pues se ha demostrado en investigaciones realizadas que una labor docente particular puede hacer la diferencia en el éxito de sus estudiantes, tal como lo afirma Darling-Hammond:

Estudiantes que están asignados a una sucesión de maestros altamente efectivos tienen ganancias significativamente mayores en los logros que los asignados a varios maestros ineficaces en secuencia, la influencia de un buen o mal profesor afecta el aprendizaje de los estudiantes no solamente en ese año sino en los años siguientes. (2006, p. 19)

La cita anterior evidencia el rol que tiene el personal docente y su notable influencia en los procesos formativos de sus estudiantes, tanto en su desarrollo, como en los aprendizajes futuros, por lo que la formación de docentes se considera como un elemento clave para la educación, para el proceso de enseñanza y aprendizaje, para el estudiantado y para la sociedad en sí misma.

El desarrollo actual de la docencia es complejo; por tanto, es importante tomar en cuenta que el concepto tradicional debe ser superado, 
pues el profesorado, a través de cursos, adquiere conocimientos teóricos que luego transmitirá a sus estudiantes. Según González y González:

La formación docente debe ser entendida como proceso educativo potenciador del desarrollo profesional del profesorado en el ejercicio de la docencia que transcurre a lo largo de la vida y que le posibilita un desempeño profesional competente, autónomo y comprometido. (2007, p. 9)

La formación docente es un proceso formativo que va más allá de los libros, de las cuatro paredes de una clase tradicional, o de los cursos aislados sin contextualización ni interdisciplinariedad; se entiende, más bien, como un proceso a lo largo de la vida en el que se aprende en la etapa de formación universitaria, pero también en la medida que se lleva a cabo el desempeño profesional, en el análisis de la labor que se realiza y en la autorreflexión y autoevaluación constante.

Es fundamental que los grupos docentes en formación puedan responder a la diversidad de estudiantes con los que se encontrarán; que favorezcan aprendizajes para la resolución de problemas; que tengan un conocimiento amplio y flexible, capaz de organizar un aprendizaje productivo, evaluar cómo, qué y para qué aprenden sus estudiantes, y que puedan adaptar la instrucción a las diferentes aproximaciones de conocimiento (Darling-Hammond, 2006). Es decir, el personal docente debe contemplar las más diversas situaciones, que incluyen desde el dominio y la mediación de conocimientos, hasta la formación para la vida, por medio de diversos acercamientos y abordajes educativos.

Se considera de gran importancia sentar las bases de un proceso de formación docente que sea realmente enriquecedor e integral, de manera que no solo brinde los conocimientos, las habilidades y las cualidades necesarias al nuevo personal educador, sino que además le permita tener experiencias prácticas más exitosas y satisfactorias. Desde esta óptica, uno de los grandes cambios que deben generarse viene desde la ubicación misma de los procesos prácticos de formación docente y la cantidad de experiencias realmente vivenciales; lo anterior para brindar experiencias significativas, contextualizadas y acordes con la realidad actual.

Por esto, se propone el modelo de formación de docentes que se lleva a cabo en Educación Comercial, carrera compartida entre la Escuela de Secretariado Profesional y la División de Educología. 


\section{El modelo de formación de docentes en Educación Comercial}

El modelo que se aplica en esta experiencia es un proceso de formación que permite una relación dialéctica entre teoría y práctica, donde, en un diálogo constante y reflexivo, la teoría desde un análisis crítico oriente la práctica; pero, a su vez, la práctica se convierta en un espacio de reflexión que luego es llevado a la clase para contrastarlo con la teoría.

Para eso, la formación de estudiantes se ejecuta con una metodología participativa que favorece teoría-práctica-reflexión-práctica; en actividades, mediante la ejecución de los módulos, se realizan prácticas en la realidad del aula ajustando la teoría en su ejecución; así como un proceso de reflexión y análisis que les permita establecer las mejoras y los ajustes necesarios a su proceso docente para su próxima sesión. El esquema del modelo del proyecto de extensión que el estudiantado realiza en las comunidades implica lo que se muestra en la figura 1.

\section{Figura 1}

\section{Esquema del modelo del proyecto de extensión}

Identificación de la

situación problema,

mediante un

diagnóstico
Diseño del plan de

acción pedagógico

para solucionar la

situación problema
Ejecución del plan de

acción pedagógico en el contexto
Valoración de los

resultados obtenidos,

mediante la reflexión y

el pensamiento crítico

Nota: Elaboración de las autoras, 2016.

Este modelo del proyecto se replica en los diversos niveles en los cuales se lleva a cabo la intervención educativa y como parte de las prácticas docentes que vivencia el estudiantado de Educación Comercial.

Estudiantes de esta carrera cumplen con dos primeros niveles de formación en los que se incluyen los cursos del área disciplinar y cursos pedagógicos. En el tercer y cuarto nivel de la carrera del Bachillerato en 
Educación Comercial continúan con cursos disciplinares base y pedagógicos, pero es en estos niveles donde se introducen las experiencias de práctica docente, como una innovación en la carrera de Educación Comercial.

Iinician sus prácticas en los cursos de tercer nivel, en los que atienden, en equipos de trabajo, a personas adultas y adultas mayores en procesos de alfabetización digital. En el cuarto año de la carrera realizan la Práctica Supervisada, en la cual orientan su experiencia al trabajo con jóvenes en situación de exclusión del sistema educativo o en riesgo social.

La figura 2 pretende mostrar los procesos de práctica docente del modelo de formación de docentes que se lleva a cabo en la carrera de Educación Comercial.

\section{Figura 2}

\section{Esquema del modelo de prácticas docentes en el modelo de formación en Educación Comercial}
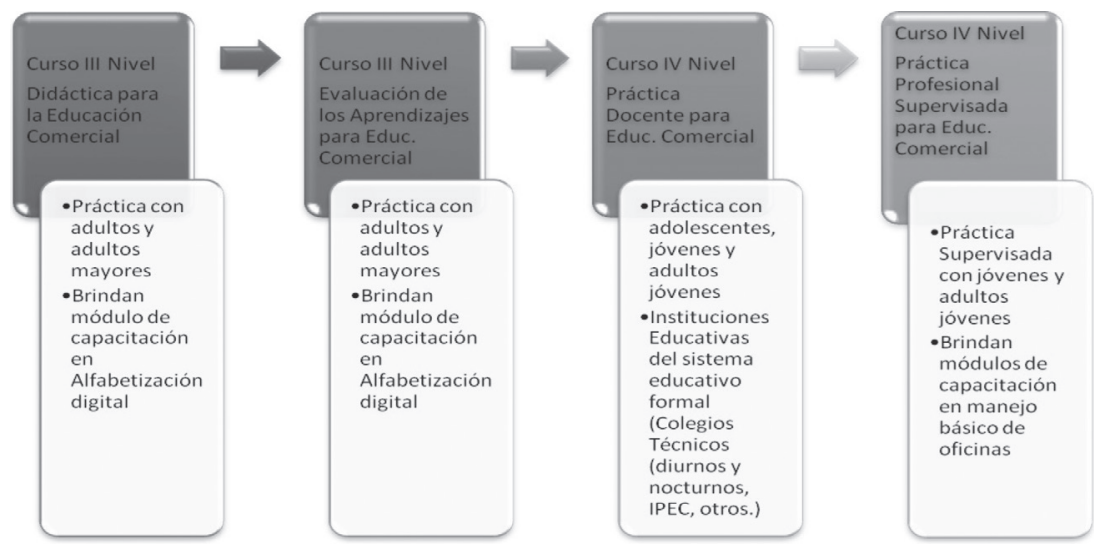

Nota: Elaboración propia de las autoras, 2016.

Como se observa en la figura 2, el proceso de las prácticas se da en forma concatenada y articulada durante los dos últimos años del bachillerato. Esto permite que el estudiantado desarrolle habilidades y capacidades pedagógicas en contextos educativos comunitarios que articulan la docencia y la extensión en su formación docente.

Por otro lado, este modelo de prácticas permite el contacto, durante su formación, de espacios educativos emergentes que le abren posibilidades de desempeño profesional como futuro profesorado en 
instituciones y organizaciones de atención a poblaciones vulnerables; así como ejercer su labor con diversas poblaciones: personas jóvenes, adultas y adultas mayores.

\section{Las intervenciones educativas}

La educación es esencial para llevar a cabo múltiples procesos y cambios en la sociedad, una de las posibilidades es la integración y aprovechamiento de las amplias oportunidades que brindan las intervenciones educativas en espacios donde se requiera un cambio para mejorar.

De acuerdo con Remedi (2004), las intervenciones educativas surgen de procesos emergentes, en los cuales se vislumbra la necesidad de cambiar o modificar algo, parcialmente o totalmente; esto es, existe algo por ser intervenido y una forma de intervenirlo, y el elemento entre ellos dos es la intervención misma. El autor señala, además, que las experiencias se relacionan estrechamente con ejecuciones prácticas orientadas a una determinada comunidad, llámese escuela, aula, ciudad, otros. Los sujetos que conforman dicho contexto entran en procesos de negociación con quien y quienes intervienen con el fin de reorientar, en este caso, una práctica formativa.

El caso que aquí se propone, Proyecto Manejo básico de oficinas tiene mayor interés en las intervenciones educativas que se orientan a influir socialmente. Tanto Corvalán y Fernández (2000) como Torres y Tenti (2000) coinciden en que las intervenciones educativas con un componente social surgen ante la determinación de una necesidad y la posibilidad de que otros entes puedan satisfacer esas necesidades. En el caso que se explicará más adelante, se detecta la necesidad que tienen poblaciones vulnerables en relación con el ámbito educativo y la posibilidad que tienen estudiantes de Educación Comercial de brindar estos procesos de enseñanza y aprendizaje.

A través de los años y principalmente con la interacción entre las ciencias sociales y educativas se van creando una serie de iniciativas y múltiples esfuerzos que se han convertido en programas y proyectos; es decir, en intervenciones educativas que buscan desde los más diversos ámbitos remediar alguna necesidad relacionada con el acceso y proceso educativo.

En el siguiente apartado se muestran algunas iniciativas sin pretender ser exhaustivas, y solo a manera de ejemplificación de las 
diversas áreas en las que se puede actuar, y las diferentes formas de buscar subsanar necesidades sociales.

La tabla 1 se basa en información generada por diversas autorías: Eming, y Fujimoto (2002), González et al. (2004), Santiago et al. (2010), Torres y Tenti (2000) y Zúñiga (2003).

\section{Tabla 1}

\section{Ejemplos de intervenciones educativas en América}

\begin{tabular}{|c|c|}
\hline PAÍS & PROGRAMA O PROYECTO \\
\hline América Latina & $\begin{array}{l}\text { Tuning-América Latina. Es una iniciativa de las universidades } \\
\text { y para las universidades que pretende iniciar un diálogo } \\
\text { para intercambiar información y mejorar la colaboración } \\
\text { entre universidades, que busca el reconocimiento entre las } \\
\text { universidades. Participan } 62 \text { universidades de } 18 \text { países } \\
\text { latinoamericanos. }\end{array}$ \\
\hline Brasil & $\begin{array}{l}\text { - Pastoral del Niño. Es una ONG que atiende más de dos millones } \\
\text { de niños y niñas mediante programas integrales que incluyen } \\
\text { alimentación, de salud y educación inicial. } \\
\text { - Un computador por estudiante. Se encarga de documentar } \\
\text { experiencias y los cambios que se han generado en los procesos } \\
\text { educativos con las computadoras. }\end{array}$ \\
\hline Chile & $\begin{array}{l}\text { - La Universidad Católica de Temuco. Brinda capacitación a } \\
\text { docentes de educación inicial en comunidades indígenas. } \\
\text { - Fundación INTEGRA. Es una ONG que trabaja con un } \\
\text { programa nacional sobre educación inicial orientado a familias de } \\
\text { bajos recursos, y apoya otros programas a nivel latinoamericano. }\end{array}$ \\
\hline Costa Rica & $\begin{array}{l}\text { - Fundación Omar Dengo. En } 1988 \text { puso en marcha el Programa } \\
\text { de Informática Educativa, con el fin de mejorar la calidad de la } \\
\text { educación apoyándose en el uso de la tecnología. } \\
\text { - TEC Digital. Es un proyecto institucional del Instituto } \\
\text { Tecnológico de Costa Rica que promueve la incorporación de las } \\
\text { TIC en las labores docentes. }\end{array}$ \\
\hline Estados Unidos & $\begin{array}{l}\text { Head Start. Surge en } 1965 \text { ante la preocupación de que los niños y } \\
\text { niñas de familias con bajos ingresos tenían menos probabilidades } \\
\text { de terminar la escuela y encontrar un trabajo estable. El programa } \\
\text { sigue liderando la educación temprana comunitaria }\end{array}$ \\
\hline México & $\begin{array}{l}\text { - Centro Infantil de Desarrollo Integral "Tierra y Libertad". Es } \\
\text { una ONG que desarrolla programas formales y no formales para } \\
\text { las poblaciones de escasos recursos. Los centros ofrecen servicios } \\
\text { nutricionales, psicológicos, pedagógicos, brindados por equipos } \\
\text { multidisciplinarios, la familia y la comunidad. } \\
\text { - Telesecundaria. Es un esfuerzo significativo para alcanzar a los } \\
\text { sectores más pobres y excluidos mediante el uso de la tecnología. }\end{array}$ \\
\hline
\end{tabular}




\begin{tabular}{|l|l|}
\hline PAÍS & PROGRAMA O PROYECTO \\
\hline Perú & $\begin{array}{l}\text { • La Universidad Católica del Perú. Promueve proyectos de } \\
\text { capacitación para maestros y maestras de educación inicial por } \\
\text { medio de la educación a distancia. } \\
\text { • Asociación Pro Educación Inicial y Básica. Es una ONG que } \\
\text { realiza estudios, investigaciones y brinda asesorías a los centros } \\
\text { educativos, a los padres y madres e instituciones académicas con } \\
\text { el fin de mejorar la calidad de los servicios. }\end{array}$ \\
\hline Venezuela & $\begin{array}{l}\text { - Centros comunitarios de aprendizaje (CECODAP). Es una ONG } \\
\text { que promueve, apoya y desarrolla acciones sobre los derechos de } \\
\text { la niñez. Garantiza y promueve espacios para su participación y } \\
\text { desarrolla proyectos educativos. }\end{array}$ \\
\hline
\end{tabular}

Nota: Sánchez (2014).

Los ejemplos presentados son una pequeña muestra de los múltiples esfuerzos que se han propuesto tanto a nivel estatal, privado, organizaciones, diversas fundaciones, $\mathrm{ONG}$, que pretenden intervenir los procesos educativos. Se muestran desde iniciativas integrales donde se consideran factores sociales, económicos, de salud y pedagógicos, hasta experiencias más concretas donde se presentan alternativas de mediación docente mediante el uso de la tecnología. También, la multiplicidad de opciones que brinda la educación para llevar a cabo intervenciones en las que se pueda impactar la sociedad y brindar alternativas de superación o solución a problemáticas ya identificadas y que requieren de acciones concretas para brindar aportes, y mejoras a la población y a las entidades.

Es de interés compartir el caso del Proyecto Manejo básico de oficinas como una intervención educativa que se ha desarrollado en la formación de docentes en Educación Comercial y con el fin de aportar soluciones educativas y formativas a poblaciones vulnerables socialmente.

\section{EI Proyecto Manejo básico de oficinas como intervención educativa}

Este proyecto nace primeramente en la Escuela de Secretariado Profesional de la Universidad Nacional, como una respuesta al compromiso de esta unidad académica con los fines y objetivos de la Universidad Nacional y su necesidad de proyectarse a la comunidad nacional. La figura 3 muestra en forma sintetizada los inicios del Proyecto. 


\section{Figura 3}

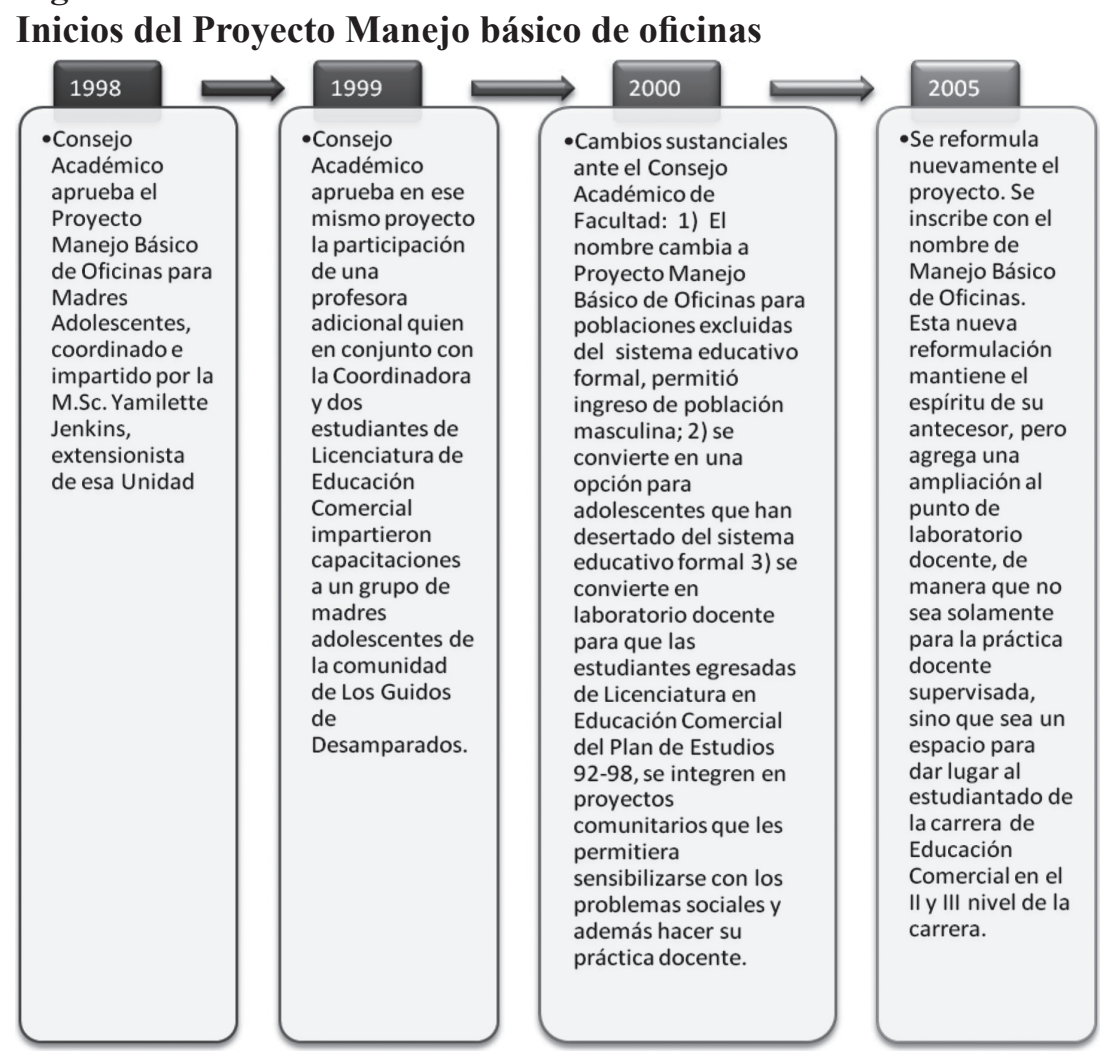

Nota: Elaboración propia de las autoras, 2017.

A partir de la reformulación del 2005, se han dado resultados significativos en cuanto a la cobertura y cantidad de participantes, comunidades atendidas e instituciones con las que se ha coordinado para su realización.

Como parte de los logros desde el 2005, se puede destacar la articulación con más de 25 instituciones o entidades que apoyan a la población atendida por el proyecto, proveniente de más de 50 comunidades, principalmente de la Gran Área Metropolitana. En su gran mayoría son personas con muy diversas problemáticas, tanto a nivel socioeconómico, académico, personal, psicológico y emocional, entre ellas: madres adolescentes; escasos recursos económicos; riesgo social; comunidades de atención prioritaria; delitos; drogas; agresión física, 
sexual y emocional; abandono; familias disfuncionales; depresión; intentos de suicidio personas excluidas del sistema educativo formal, menores de edad que trabajan, entre otros.

Se han atendido más de 950 adolescentes y jóvenes a quienes se ha capacitado en manejo básico de oficinas y, desde el 2009, más de 100 personas mayores de treinta años han sido capacitadas en el uso básico de la computadora y las tecnologías de la información y comunicación.

Respecto a la participación de estudiantes de la carrera de Educación Comercial, desde el 2005 hasta el 2014, han participado 130 del nivel de bachillerato, con un promedio anual de 13 a 14. A partir del 2009 se integran estudiantes del III nivel de la carrera para un total de 60 estudiantes.

Desde el 2005, en el proyecto han participado permanentemente dos académicas, una de la Escuela de Secretariado Profesional y otra de la División de Educología. En el 2009 se incorpora oficialmente como unidad participante conjunta del Proyecto la División de Educología; a pesar de que desde 2007 venía involucrándose de manera directa por medio de los cursos del componente pedagógico, donde se realizaban las prácticas con personas adultas mayores.

De acuerdo con la formulación 2013-2015, el proyecto tiene como objetivo general: Desarrollar procesos académicos de articulación de la docencia y la extensión en la carrera de Educación Comercial que favorezcan la formación de personal formador con acciones más prácticas que promuevan la atención a poblaciones en desigualdad de oportunidades.

Con base en lo indicado por Jordán et al. (2011) respecto a que una intervención educativa es entendida como un conjunto de acciones de carácter pedagógico, motivacional u otro, que son llevadas a cabo por agentes de intervención, a partir de un programa diseñado previamente y con la intención de que quienes reciban la intervención alcancen lo propuesto, además, del objetivo y acciones del Proyecto, se puede indicar que este se ajusta a lo que toda intervención educativa debe pretender; tal y como se representa en la figura 4. 


\section{Figura 4}

\section{Componentes de una intervención educativa y su cumplimiento por el Proyecto}

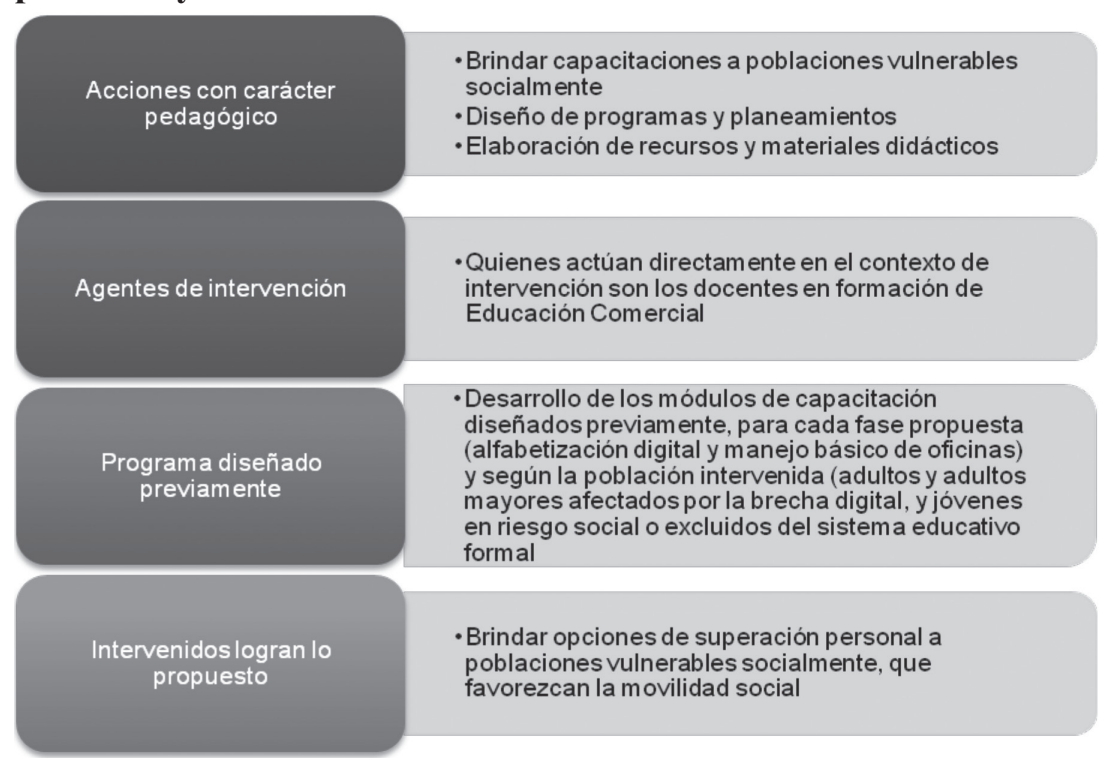

Nota: Elaboración propia de las autoras, 2017.

De acuerdo con la figura 4, el Proyecto cumple con lo definido como intervención educativa, desde el diseño o planeamiento previo de las acciones pedagógicas que se llevarán a cabo, hasta tener definido quiénes llevan a cabo la intervención, a quienes se interviene y con qué intención.

Además, el proyecto actúa en doble vía, en un mutuo-aprendizaje, ya que docentes en formación fortalecen y amplían sus procesos de práctica, lo que les permite interactuar con diferentes poblaciones, con necesidades educativas y personales muy diversas; por lo que también se favorecen cambios a nivel pedagógico, didáctico y personal para el futuro personal docente.

En este caso y de acuerdo con lo desarrollado anteriormente, el Proyecto como intervención educativa favorece cambios, tanto a las poblaciones atendidas, como al profesorado en formación. Esto se evidencia en los aportes que se presentan a continuación. 


\section{Aportes del proyecto como intervención educativa}

A partir de la intervención educativa que se efectúa con este proyecto, se pueden observar los aportes que se generan en las necesidades atendidas en la atención de esas poblaciones en desigualdad y en los procesos de formación docente.

Primeramente, se enumeran las necesidades atendidas en las poblaciones a quienes se dirige la intervención educativa:

1. Se crean espacios de educación no formal en las comunidades, que se convierten en un vínculo entre la universidad y el contexto social, permiten la realimentación entre ambos elementos, su interrelación y mutuo fortalecimiento.

2. Se brinda capacitación a juventud excluida del sistema educativo formal y en riesgo social, para que adquieran conocimientos, destrezas y habilidades, tanto técnicas como humanas, que le permitan integrarse a un puesto de trabajo en el área secretarial a nivel básico y que, con ello, se puedan generar procesos de movilidad social.

3. Se promueve la inclusión educativa y social al proporcionar una capacitación práctica relacionada con el trabajo; es decir, impacta la vida de las personas y logra sus objetivos esenciales como es la formación de jóvenes vulnerables socialmente.

4. Se favorece, que los grupos de jóvenes que se atienden encuentren, en los módulos que se imparten, un elemento motivador para su reinserción en el sistema educativo, que les permita concluir sus estudios formales y continuar en su proceso de fortalecimiento, crecimiento personal y profesional.

5. Se impulsa la disminución de la brecha digital, al brindar a personas adultas mayores de treinta años, que no han tenido contacto con el uso de la tecnología y en muchos casos ni siquiera han tenido la experiencia de lo que significa el uso de una computadora, la oportunidad de contar con una formación básica con el uso de la computadora, las tecnologías de la información y la comunicación (Windows, paquetes de Office, internet y digitación). 
Sin embargo, no solo los sujetos participantes de las diversas comunidades se benefician con los módulos que se ejecutan mediante el proyecto, sino también se generan cambios en el estudiantado universitario con este proceso.

Seguidamente, se mencionan los elementos que, desde la formación docente de estudiantes de la carrera de Educación Comercial, esta intervención educativa favorece:

1. La interrelación entre la docencia y la extensión universitaria se ve reflejada en este caso en la articulación entre un proyecto de extensión y la formación docente, la cual permite contar con contextos que complementan y fortalecen el proceso de formación académica.

2. La articulación de la docencia y la extensión fortalece la sensibilidad social al generar experiencias solidarias, inclusivas, democráticas y autónomas, para la vida profesional y personal de docentes en formación.

3. El Proyecto Manejo básico de oficinas se convierte en ente articulador entre el componente pedagógico, la disciplina y la comunidad, como se refleja en la figura 5.

\section{Figura 5}

\section{Articulación docencia y extensión mediante proyecto}

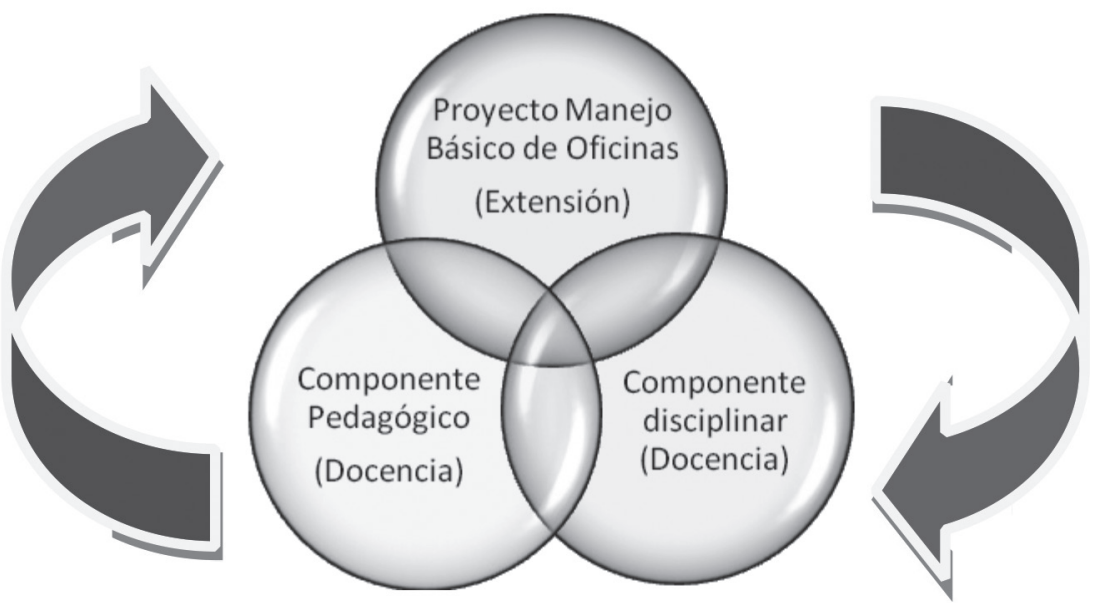

Nota: Elaboración propia de las autoras, 2016. 
4. Vincula la formación docente inicial con la extensión universitaria, de manera que desde los cursos de formación pedagógica se implementan procesos de prácticas. Desde los primeros años el estudiantado es responsable del desarrollo de cursos de educación no formal que ofrece el proyecto.

5. Se rompe con el esquema de enfocarse solo en la secundaria del sistema educativo del país; se generan espacios de prácticas en diferentes contextos, en particular el comunitario, con poblaciones diversas que incluyen personas adolescentes, jóvenes adultas y adultas mayores; en diferentes situaciones sociales, lo que les permite una visión más amplia para su futuro desempeño profesional.

6. Se brindan procesos prácticos desde la formación inicial, que anticipan las experiencias prácticas formales y amplían la cantidad de vivencias en el campo, con lo cual se fomenta la reflexión y el pensamiento crítico.

7. Se logra, así, vincular el proyecto Manejo básico con la gestión curricular del plan de estudios de la carrera de Educación Comercial y mantener una constante actualización metodológica del currículo de la carrera.

8. Esta intervención educativa permite al estudiantado, en su proceso de formación docente, fortalecer la capacidad de toma de decisiones, mejorar su desempeño docente, adaptarse a diversidad de grupos etarios, desarrollar cualidades docentes, fortalecer sus habilidades sociales y la resolución de problemas in situ, mediante experiencias formativas contextualizadas.

9. Fortalece el desarrollo de actitudesy valores comoresponsabilidad, trabajo en equipo, tolerancia, comprensión, empatía, solidaridad, confianza en sí, y sensibilidad social, entre otras. Lo anterior evidencia una formación más integral del estudiantado, mediante las prácticas realizadas.

Por estas razones, el Proyecto como intervención educativa realmente genera aportes y cambios, tanto a las poblaciones atendidas como a quienes llevan a cabo el proceso educativo. 


\section{Conclusiones}

Se evidencia la importancia que tiene una iniciativa como el Proyecto Manejo básico de oficinas, el cual, como intervención educativa, ha permitido innovar el currículo de formación docente y, por medio de la educación, impactar el contexto con la atención de necesidades sociales y educativas, mediante un proyecto de extensión y docencia.

El estudiantado en su formación valora ampliamente este tipo de experiencias. La siguiente expresión resume el sentido que le confiere a este tipo de prácticas: "Creo y considero que es necesario que este tipo de prácticas sean empleadas con mayor regularidad en la Universidad, porque el estudiante se familiariza más con su profesión" (Estudiante Educación Comercial). Por lo tanto, el modelo como tal es pertinente para la formación de personal formador, pues este, a lo largo de la carrera, adquiere un sentido más práctico en su formación, en contraposición a modelos donde se brinda una práctica profesional supervisada al final de su plan de estudios.

Como se mencionó en este escrito, el Proyecto favorece la articulación de la docencia y la extensión y la flexibilización del currículo, al contextualizarlo y ubicarlo en las comunidades, donde existen múltiples necesidades educativas. Esto lo hace más práctico, más vivencial y significativo, es decir, propone mejoras para innovar la formación de docentes, desde la práctica misma y las experiencias que trascienden las aulas, lo cual solo se logra mediante el contacto directo con las poblaciones, en este caso, con las personas jóvenes, adultas y adultas mayores, que la sociedad misma ha excluido de oportunidades de aprendizaje para la vida, y que encuentran en este proyecto un espacio de crecimiento y motivación para seguir adelante.

El contexto social brinda aprendizajes al futuro profesorado, por lo que plantear intervenciones educativas que contribuyan a solventar determinadas necesidades y al mejoramiento social, se convierte en un vínculo más que necesario para el logro de la formación de profesionales que realmente cumplan el sentido de la universidad necesaria. 


\section{Referencias}

Corvalán, J. y Fernández, G. (2000). Apuntes para el análisis de la participación en experiencias educativas y sociales. Revista Latinoamericana de Estudios Educativos, 30(4), 9-50.

Darling-Hammond. (2006). Powerful Teacher Education. San Francisco, CA: Jossey-Bass a Wiley Imprint.

Eming, M. y Fujimoto, G. (2002). Desarrollo infantil temprano: Lecciones de los programas no formales. OEA- BID.

Godorokin, I. (2005. La formación docente y su relación con la epistemología. Revista Iberoamericana de Educación, 37(5), 2.

González, J. y otros. (2004). Tuning-América Latina: un proyecto de las universidades. Revista Iberoamericana de Educación, 35, 151-164.

González, R. y González, V. (2007). Diagnóstico de necesidades y estrategias de formación docente en las universidades. Revista Iberoamericana de Educación, 43, 2-9.

Pogré, P. (2004). Los temas en cuestión. UNESCO/OREALC - PROEDUCA/GTZ, 120.

Remedi, E. (2004). De licenciado a maestro: Tiempos completos y certificados. Memorias de Congreso. México: CESU-UNAM.

Sánchez, I. (2014) Examen de síntesis. Doctorat en Sciences de l'Education. Université de Montréal. Canadá.

Santiago, A., Severin, E., Cristia, J., Ibarrarán, P., Thompson, J. y Cueto, S. (2010). Evaluación Experimental del Programa "Una Laptop por Niño" en Perú. Aportes. No. 5, 1-12.

Torres, R. y Tenti, E. (2000). Políticas educativas y equidad en México: La experiencia de la educación comunitaria, la telesecundaria y los programas compensatorios. SEP-IIPE UNESCO.

Universidad Nacional de Costa Rica. (2013-2015). Proyecto de Extensión manejo básico de oficinas (Formulación). Heredia: Autor.

Universidad Nacional de Costa Rica. (2016). Pagina web. Recuperado de http://www.una.ac.cr/

Zúñiga, M. (2003). Aprendizaje mediado por tecnologías digitales: La experiencia de Costa Rica. Educación y nuevas tecnologías. Experiencias en América Latina IIPE-UNESCO. 